\title{
International Journal of
}

\section{Clinical Obstetrics and Gynaecology}

ISSN (P): 2522-6614

ISSN (E): 2522-6622

(C) Gynaecology Journal

www.gynaecologyjournal.com

2021; 5(1): 432-434

Received: 26-11-2020

Accepted: 29-12-2020

\section{Dr. Anandita}

Department of Gynecological Oncology, Amrita Instituto of Medical Science, Kochi, Kerala, India

Dr. Anupama Rajanbabu

Obstetrics and Gynaecology,

MRCOG, Department of

gynaecological oncology, Amrita

Institute of Medical Sciences,

Kochi, Kerala, India

Dr. Indu R Nair

Department of Pathology, Amrita Institute of Medical Sciences,

Kochi, Kerala, India

Dr. Pavithran K

Department of Medical Oncology,

Amrita Institute of Medical

Sciences, Kochi, Kerala, India

Corresponding Author:

Dr. Anandita

Department of Gynecological Oncology, Amrita Instituto of Medical Science, Kochi, Kerala, India

\section{Unusual presentation of an endometrial stromal sarcoma: A case report}

\author{
Dr. Anandita, Dr. Anupama Rajanbabu, Dr. Indu $R$ Nair and Dr. \\ Pavithran K
}

DOI: https://doi.org/10.33545/gynae.2021.v5.i1g.852

\section{Abstract}

Endometrial stromal sarcomas are the rare tumor accounting for $<2 \%$ of all uterine tumor. It is diagnosed mostly in 40-55 years age with slow growth with extrauterine spread in $40-50 \%$ of the cases at the time of diagnosis. In this case report, ESS was diagnosed in a 40 year old lady with pulmonary and cardiac metastasis surgically managed followed by adjuvant hormonal treatment with local recurrence treated with radiotherapy with good prognosis. ESS with distant metastasis can have better prognosis if treated surgically with complete resection and adjuvant therapy with multidisciplinary approach and regular follow up.

Keywords: Endometrial stromal sarcoma, distant metastasis, adjuvant treatment

\section{Introduction}

Endometrial stromal sarcomas are the rare tumor accounting for $<2 \%$ of all uterine tumor. According to clinical, pathological and genetic characteristics, the Fourth Edition of the World Health Organization classification in $2014^{[1]}$ divided endometrial stromal tumors into the following four categories: Endometrial stromal nodule, undifferentiated uterine sarcoma, highgrade endometrial stromal sarcoma (ESS) and low-grade ESS. It is diagnosed mostly in 40-55 years age with slow growth with extrauterine spread in $40-50 \%$ of the cases at the time of diagnosis ${ }^{[2]}$. Herein reported is a case of low grade ESS presenting 5 years after a hysterectomy done for benign disease.

\section{Case Report}

A 40-year-old lady with history of Total Abdominal Hysterectomy + Bilateral salphingoophrectomy in 2008 for Abnormal uterine bleeding -leiomyoma presented to local hospital with abdominal pain and swelling in 2013. Her Ultrasound scan showed large heterogenous solid - cystic lesion in right adnexa, suggestive of malignant ovarian mass and enlarged left ovary with large solid areas with CA-125 being 59.4. She was evaluated with CT Scan which was suggestive of Malignant Right ovarian tumor (Figure 1) with regional lymphadenopathy and lung metastasis (Figure 2) with separate moderately enhancing lesion posterior to bladder on the left side. FAC from the mass showed malignant cells and she was managed at a teritiary care hospital where she received chemotherapy with 6 cycles of Paclitaxel and Carboplatin. As there was no response to chemotherapy, she was referred to our institute. Here, on evaluation she was found to have right atrial mass $(3.6 \times 3.9) \mathrm{cm}$ (Figure 3$)$ extending to IVC? Thrombus? Metastasis.

As there was no response to the initial chemotherapy and patient had great physical discomfort due to the abdominal mass, palliative surgery was planned for symptom relief. Intraoperatively a multicystic mass with solid components present about $25 \times 20 \mathrm{~cm}$ with bilateral ovarian masses of $10 x 10 \mathrm{~cm}$ each. She underwent palliative surgery in September 2014. Histopathology report showed Cellular spindle cell neoplasm, involving both ovaries with Omentum. Intraabdominal deposit, infundibulopelvic ligament and vaginal vault tumor deposit with two right iliac nodes being positive for metastasis. Immunohistochemistry was positive for CD10, negative for SMA, Inhibin and ER-90\%show moderate nuclear intensity and PR-95\% show moderate to intense nuclear positivity suggestive of Endometrial stromal sarcoma-low grade. She was started on Adjuvant therapy with T letrozole. 
Right atrial thrombus was excised in a second sititing after 6 months with histopathology reported as hyalinised tissue partly lined by endothelial cells. Chest CT showed regression of the pulmonary lesions with letrozole.

She was on regular follow up and after a DFI of 4 years she was diagnosed to have a pelvic recurrence. She received RT $50 \mathrm{~Gy} / 20 \#$ to tumor alone with, margin getting 45Gy/20\# completed on august 2019 and Post RT after 4 months the lesion regressed in size. Presently patient is asymptomatic, doing well on regular follow up.

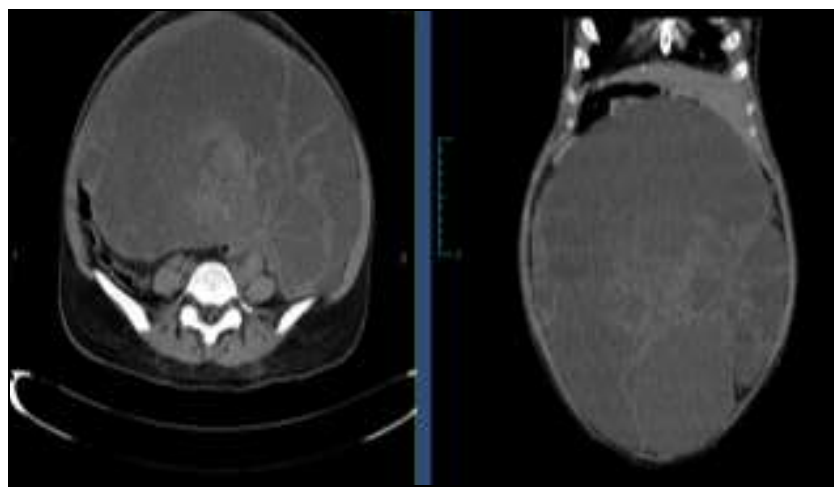

Fig 1: Axial and coronal sections showing abdominopelvic mass

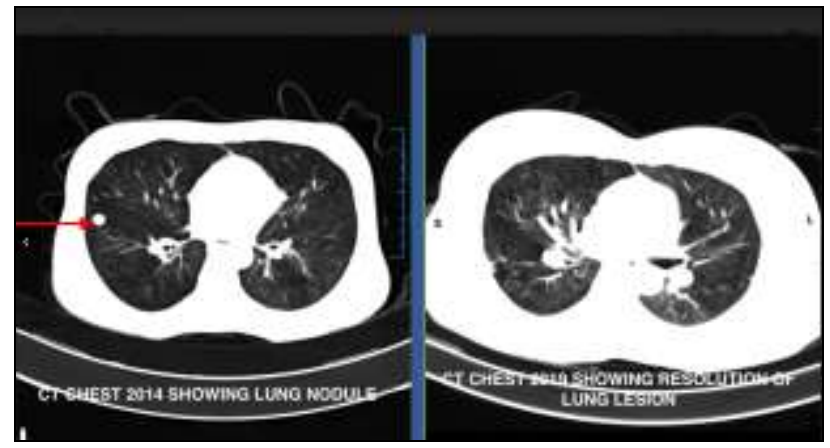

Fig 2: A showing lung lesion and Fig 2 B showing resolution of the lung lesion

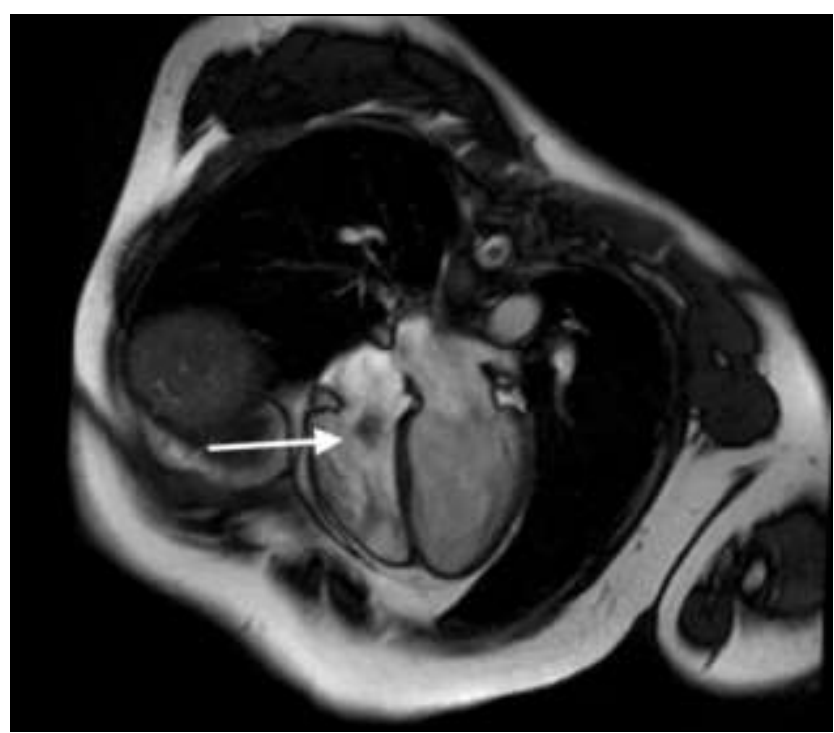

Fig 3: Showing right atrial thrombus

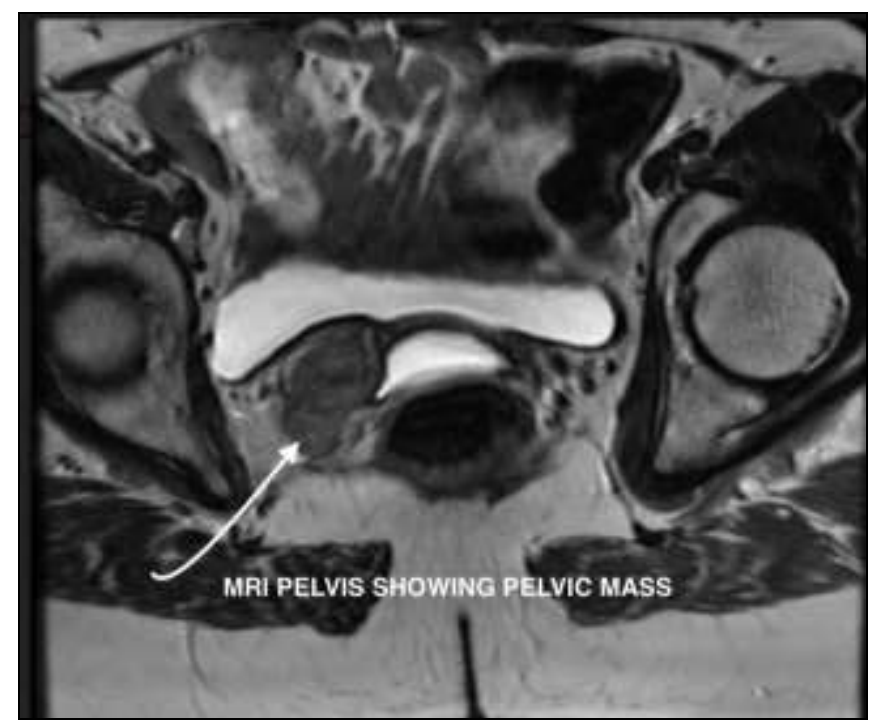

Fig 4: Showing recurrence

\section{Discussion}

The annual incidence of ESS is 1-2 per million women. ESS is an indolent tumor with local recurrences and distant metastasis can occur even 20 years after initial diagnosis. Immunohistochemistry plays an important role in the diagnosis of ESS. CD10 expression has been shown in several nonhematopoietic neoplasm, including endometrial stromal sarcomas. ESS is almost always positive for both estrogen and progesterone receptors. ESS is staged according to the FIGO staging and prognosis depends upon the stage but out of all sarcoms ESS has better life expectancy.

Surgery represents the most important procedure in the management of patients with ESS. The role of adjuvant therapy lie in the prevention and treatment of recurrent, residual and metastatic lesions. Aromatase inhibitors and progestins ate the most common hormonal agents used in the treatment of ESS. Radiotherapy is useful in the control of local recurrence.

In most of the literature it has been seen that in patients with infiltration of IVC, RA, and RV, the best option is extensive surgery.

In a case report and literature review by ${ }^{[3]} \mathrm{Xu} \mathrm{Y}$ etal demonstrated that pulmonary metastases of low-grade ESS are not common but should be disregarded. The clinical manifestations are not specific and diagnosis is often difficult. The combination of clinical history, imaging results and histological findings is essential for the diagnosis of low-grade ESS with pulmonary metastasis.

A combination of surgery and adjuvant therapy may improve the treatment outcome.

In a study of retrospective analysis of factors affecting recurrence of endometrial stromal sarcoma by Agarwal et al concluded that Incomplete surgery and no adjuvant treatment in ESS are associated with poor DFS. Complete staging surgery is associated with improved DFS. Resection of recurrent disease is associated with survival advantage ${ }^{[4]}$.

Shalerian et al reported a case report with heart and lung metastasis of ESS in a40 year old lady and reported that surgical approach in such case offers the possibility of an excellent longterm prognosis ${ }^{[5]}$. 
Tadic et al have reported a case report of ESS with right heart mass in a 61 year old who had been treated 10 years before for low-grade endometrial stromal sarcoma by hysterectomy and adnexectomy followed by hormone-and radio-therapy and highlights the importance of a multidisciplinary approach in managing patients with ESS, and the necessity for regular follow-up CT scans even 5, 10, or 20 years after initial diagnosis.

In this case report, ESS was diagnosed in a 40 year old lady with pulmonary and cardiac metastasis surgically managed followed by adjuvant hormonal treatment with local recurrence treated with radiotherapy with good prognosis.

Hence, we conclude that ESS with distant metastasis can have better prognosis if treated surgically with complete resection and adjuvant therapy with multidisciplinary approach and regular follow up.

\section{References}

1. Ali RH, Rouzbahman M: Endometrial stromal tumours revisited: An update based on the 2014 WHO classification. J Clin Pathol 2015;68:325-332.

2. Xue WC, Cheung AN. Endometrial stromal sarcoma of uterus. Best Pract Res Clin Obstet Gynaecol 2011;25:719732.

3. Xu Y, Liang Z, Guo J, Su X, Lu Y, Guan X, et al. Cystic and solitary nodular pulmonary metastases in a patient with low-grade endometrial stromal sarcoma: A case report and literature review". Oncology Letters 2019;18(2):1133-1144.

4. Agarwal R, Rajanbabu A, Nair IR, Satish C, Jose G, Unikrishnan UG, et al. Endometrial stromal sarcoma-A retropsective analysis of factors affecting recurrence. Eur J Obstet Gynecol Reprod Biol 2017;216:92-97.

5. Shakerian B, Mandegar MH, Moradi B, Roshanali F. Heart and Lung Metastases From Endometrial Stromal Sarcoma in a Forty-Two-Year-Old Woman. Research in cardiovascular medicine 2015;4(3):e26066.

6. Tadic M, Belyavskiy E, Cuspidi C, Pieske B, Haßfeld S. Right heart masses in a patient with endometrial stromal sarcoma. Journal of Clinical Ultrasound 2019. 